\title{
Palmar-plantar erythema associated with combination chemotherapy
}

\author{
A. Pagliuca, R. Kaczmarski and G.J. Mufti \\ Academic Department of Haematological Medicine, King's College Hospital, Denmark Hill, \\ London SE5 $8 R X, U K$.
}

\begin{abstract}
Summary: We report three cases of a distinctive palmar-plantar erythema associated with the treatment of non-Hodgkin's lymphoma and acute myeloid leukaemia. The rash is characterized by a painful, sharply demarcated, intense erythema of the palms and/or soles followed by bulla formation, desquamation and healing. The eruption is self limiting in nature and did not adversely affect prognosis. Treatment need be neither altered nor stopped; only symptomatic measures are required.
\end{abstract}

\section{Introduction}

Skin disorders are a common diagnostic problem in patients undergoing chemotherapy for haematological and other malignancies. With the advent of intensive combination chemotherapy it may be difficult to identify which is the causative drug especially when the patient is on other medications such as antibiotics. The normal course of action would be to stop the causative agent and consider rechallenge; however, this may compromise therapy and the eventual outcome. We report three cases of distinctive painful erythema involving the palms and soles in patients receiving combination chemotherapy, in whom treatment was neither reduced nor stopped.

\section{Case reports}

\section{Case 1}

A 27 year old man presented with stage IVB immunoblastic lymphoma and was treated with MACOP-B. ${ }^{1}$ This regimen consists of alternating weekly cycles of methotrexate (with folinic acid rescue) and vincristine (weeks 2, 6 and 10), adriamycin and cyclophosphamide (weeks 1, 3, 5, 7,9 and 11) and vincristine and bleomycin (weeks 4, 8 and 12). Prednisolone and co-trimoxazole were given throughout treatment.

After the first dose of methotrexate (total dose $700 \mathrm{mg}$ ) he developed mucositis and dysaesthesiae in his hands and feet which resolved over 72 hours. He proceeded with treatment as scheduled and developed minimal mucositis with his second dose

Correspondence: G.J. Mufti, M.R.C.Path.

Accepted: 7 August 1989 of methotrexate. Treatment weeks 7,8 were uneventful but 4 days after having week 9 of treatment he suddenly developed a well demarcated, acutely tender erythematous rash on the palms, fingers and soles. Oedema was marked, restricting fine movements of the fingers and he found it difficult to walk. The rash became bullous and started desquamating 10 days later. He developed finger nail dystrophy and then lost his nails. It was suspected that the rash was methotrexate-induced so week 10 of treatment was switched with week 12. No dose reductions were made and he had his final dose of methotrexate without recurrence of the erythema. Skin healing occurred without scarring and management was purely supportive with analgesia and emulsifying ointment. He is presently in remission.

\section{Case 2}

A 60 year old woman presented with stage IVB cell centroblastic lymphoma and was treated with MACOP-B. ${ }^{1}$ Treatment was uneventful other than mild mucositis with week 2 of treatment; however, 6 hours after having her second course of methotrexate (dose $600 \mathrm{mg}$ ) she developed severe itching in her palms and soles associated with erythema and oedema (Figure 1). The rash became bullous on her hands and desquamated without scarring 9 days later. The third course of treatment with methotrexate was given as scheduled with no recurrence of the skin rash.

\section{Case 3}

A 49 year old woman with acute myeloid leukaemia was treated with daunorubicin $50 \mathrm{mg} / \mathrm{m}^{2}$ intravenously days 1,3 and 5 , cytosine arabinoside 


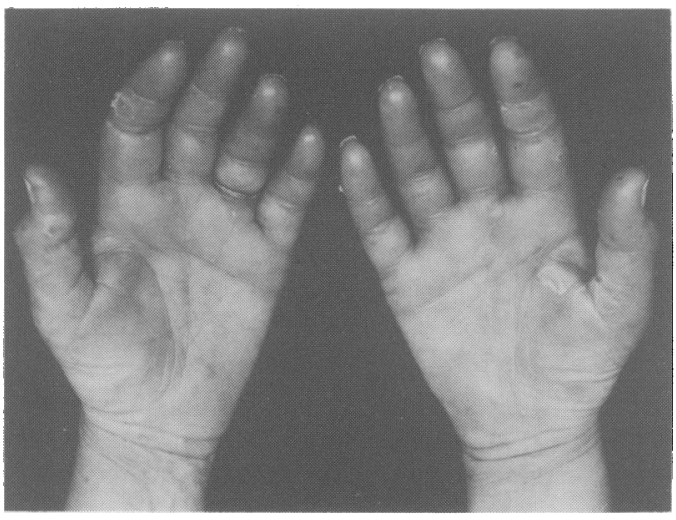

Figure 1 Case 2: palmar erythema, oedema and desquamation with demarcation at the wrist.

$100 \mathrm{mg} / \mathrm{m}^{2}$ intravenously every 12 hours for 10 days and 6-thioguanine $100 \mathrm{mg} / \mathrm{m}^{2}$ orally, twice daily for 10 days. During her induction chemotherapy she developed a painful erythematous rash involving her hands on the final day of treatment. There was minimal oedema but no bullae. The rash became itchy and desquamated over the next 14 days. The rash recurred with the following courses of maintenance therapy but no treatment modification was made.

\section{Discussion}

Toxic erythema of the palms and soles has only recently been described in patients undergoing cytotoxic chemotherapy. A variety of diagnostic labels have been used but they all describe a similar clinical picture. ${ }^{2-6} \mathrm{~A}$ prodrome of dysaesthesiae may develop in the palms and soles which is characteristically followed by the sudden onset of a well demarcated, acutely tender erythematous rash on the palms, fingers and soles, often associated with oedema. The rash may become bullous and then desquamate without scarring.

\section{References}

1. Klimo, P. \& Connors, J.M. MACOP-B chemotherapy for the treatment of diffuse large-cell lymphoma. Ann Intern Med 1985, 102: 596-602.

2. Baer, M.R., King, L.E. \& Wollf, S.N. Palmar-plantar erythrodysesthesia and cytarabine. Ann Intern Med 1985, 102: 556

3. Shall, L., Lucas, G.S., Whittaker, J.A. \& Holt, P.J.A. Painful red hands: a side effect of leukaemia therapy. $\mathrm{Br} J$ Dermatol 1988, 119: 249-253.

4. Cox, Robertson, D.B. Toxic erythema of palms and soles associated with high-dose mercaptopurine chemotherapy. Arch Dermatol 1986, 122: 1413-1414.

5. Lokich, J.J. \& Moore, C. Chemotherapy-associated palmarplantar erythrodysesthesia syndrome. Ann Intern Med 1984, 101: $798-800$.
A similar reaction has been reported with cytosine arabinoside, either as a single agent or in combination therapy, ${ }^{2,3}$ mercaptopurine,${ }^{4}$ doxorubicin, ${ }^{5} 5$-fluorouracil ${ }^{5}$ and methotrexate as part of the ProMACE protocol. ${ }^{6}$

Localization of skin toxicity to the palms and soles is an interesting feature and factors involved could be rapid cell division at these sites, gravitational forces, temperature gradients and vascular anatomy peculiar to the palms and soles. Previous reports have pointed to a toxic rather than an allergic reaction. ${ }^{6}$ In cases 1 and 2 recurrence did not occur with rechallenge and in case 3 the rash only occurred after the final day of treatment pointing to a cumulative toxic response rather than an allergic phenomenon.

It is difficult to be sure which drugs caused this reaction. However, in view of the temporal relationship one might suggest methotrexate in cases 1 and 2 and cytosine arabinoside in case 3 . A toxic interaction of several of the agents cannot be excluded and a blistering reaction beneath pressure points and callus sites of the hands and feet has been reported with MACOP-B. ${ }^{\prime}$ This was considered to represent a toxic interaction between bleomycin and other drugs. Bleomycin is known to concentrate in the skin and has numerous cutaneous side effects including hyperpigmentation, sclerosis, oedema and Raynaud's phenomenon, ${ }^{7,8}$ and may have been a co-factor with cases 1 and 2 .

It is important that physicians recognize this distinct clinical entity and its possible causative drugs so that reassurance can be offered to the patient. Although dramatic in presentation the eruption is self limiting and normally requires only symptomatic treatment. Systemic corticosteroids have been reported to be effective, when given early, in cases caused by cytosine arabinoside. ${ }^{2}$ In conclusion, withdrawal or reduction of chemotherapy which may jeopardise treatment is not always necessary.

6. Doyle, L.A., Berg, C., Bottino, G. \& Chabner, B. Erythema and desquamation after high dose methotrexate. Ann Intern Med 1983, 98: 611-612.

7. Cohen, I.S., Mosher, M.B., O’Keefe, E.J., Klaus, S.N. \& De Conti, R.C. Cutaneous toxicity of bleomycin therapy. Arch Dermatol 1973, 107: 553-555.

8. Vogelzang, N.J., Bosl, G.J., Johnson, K. \& Kennedy, B.J. Raynaud's phenomenon: a common toxicity after combination chemotherapy for testicular cancer. Ann Intern Med 1981, 95: $288-292$. 